\title{
Simultaneous measurement of temperature, density and velocity in gas flows by modulated photoluminescence
}

\author{
J.-B. Liu, C.-S. Liu and J.-R. Shi \\ Institute of Mechanics, Chinese Academy of Sciences, P.O. Box 2251, Beijing 100080, P.R. China
}

\begin{abstract}
A novel possibility to determine the temperature, density and velocity simultaneously in gas flows by measuring the average value, amplitude of modulation and phase shift of the photoluminescence excited by a temporally or spatially modulated light source is investigated. Time-dependent equations taking the flow, diffusion, excitation and decay into account are solved analytically. Different experimental arrangements are proposed. Measurements of velocity with two components, and temporal and spatial resolutions in the measurements are investigated. Numerical examples are given for $N_{2}$ with biacetyl as the seed gas. Practical considerations for the measurements and the relation between this method and some existing methods of lifetime measurement are discussed.
\end{abstract}

\section{Introduction}

Many conventional methods of flow field diagnostics measure directly only one parameter of the gas flow. For example, interferometry measures directly only the density $N$, Doppler velocimetry directly measures only the velocity $V$, etc. Although these techniques are very successful, measurement of only one parameter is usually not enough for a thorough investigation of the gas flow. For investigating the correlation between some quantities, two parameters should be measured simultaneously. For a complete description of a gas flow with constant and homogeneous chemical composition, three parameters, temperature $T$, density $N$ and velocity $V$, must be measured simultaneously. Therefore one of the present trends in the development of flow field diagnostics is to measure two or three parameters simultaneously.

There are several methods for simultaneous measurements of two parameters. The most common is to use two different kinds of instruments simultaneously, each for the measurement of one parameter. For example, to simultaneously measure $T$ and $V$, the combinations of CARS and LDA (Goss et al. 1984, 1988; Fujii et al. 1983, 1984), thermocouple and LDV (Stephered et al. 1982; Bram et al. 1983), photothermal and photoacoustic deflections (Rose and Gupta 1986) were used. Some techniques can measure two parameters simultaneously. For example, laser induced fluorescence (Gross et al. 1987), spontaneous Raman scattering
(Blint et al. 1980; Smith 1980 a, b), laser absorption spectroscopy (Luck and Thielen 1978), electron-beam fluorescence (Smith and Driscoll 1975) were used to simultaneously measure both $T$ and $N$. There are only a few techniques which can measure three parameters ( $T, N$ and $V$ ) simultaneously; these include resonant Doppler velocimetry (Cheng et al. 1983 a; Miles 1975; Miles et al. 1978; Zimmermann and Miles 1980), laser induced fluorescence (Cheng et al. 1983 b) and laser induced biacetyl phosphorescence (Liu et al. 1988).

In this paper, possible ways to measure $T, N$ and $V$ simultaneously by measuring the average value, amplitude of modulation and phase shift of the photoluminescence excited by a temporally or spatially modulated light source are investigated (Sect. 2). Time-dependent equations taking the flow, diffusion, excitation and decay effects into account are solved analytically (Sect. 3). Different possible experimental arrangements are proposed (Sect. 3). Measurements of velocity with two components (Sects. 4 and 5), and temporal and spatial resolutions in the measurements (Sect. 6) are investigated. Numerical examples are given for $\mathrm{N}_{2}$ with biacetyl as the seed gas. Practical considerations and relations between this method and some existing methods of lifetime measurement are discussed (Sect. 7).

\section{Model}

Consider a flow field diagnostics experiment as shown in Fig. 1 . The primary gas (e.g. $N_{2}$ ) premixed homogeneously with some kind of gaseous photoluminescent material (e.g. biacetyl vapour) flows along the $x$ axis. The mol fraction of the photoluminescent gas is assumed to be constant everywhere. A parallel beam from the light source with appropriate spectrum is focused into a thin sheet on the $x y$ plane by a cylindrical lens. The intensity $I_{s}$ of this light sheet is assumed to be uniform on the $x y$ plane and varying cosinusoidally with time $t$.

$I_{s}(t)=c_{s}\left(1-m_{t} \cos (2 \pi \omega t)\right) \quad 0 \leq m_{t} \leq 1$ 


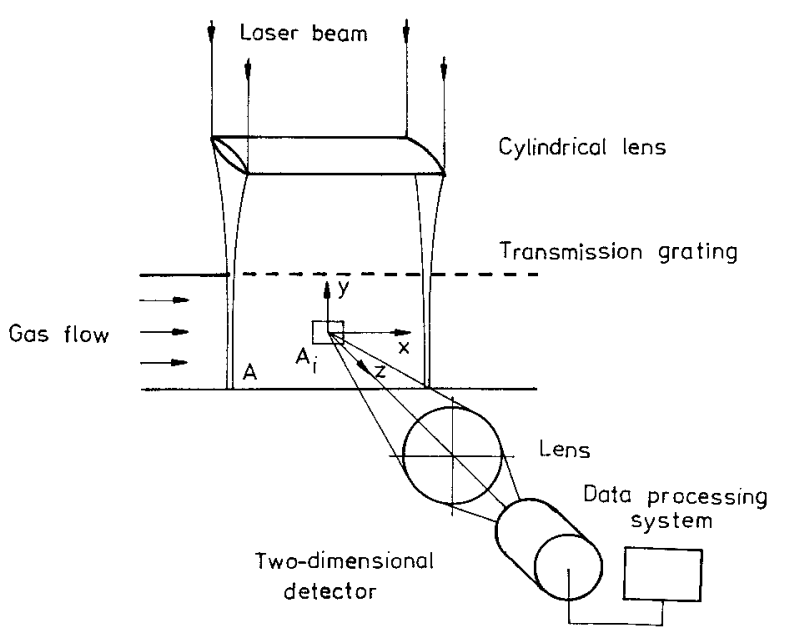

Fig. 1. Scheme of experimental technique for simultaneous measurements of temperature, density and velocity by modulated photoluminescence

where $c_{s}$ is the average intensity, which is a constant, $m_{t}$ the peak to peak change of the intensity normalized by $c_{s}, \omega$ the frequency. The light sheet irradiates the gas flow through a transmission grating, the transmittance $T(x)$ of which is zero in the region $x \leq 0$ and varies cosinusoidally in the region $x>0$

$T(x)=\left\{\begin{array}{ll}0 & x \leq 0 \\ \frac{1}{2}\left(1-m_{s} \cos (2 \pi f x)\right) & x>0\end{array} \quad 0 \leq m_{s} \leq 1\right.$

where the $m_{s}$ is the peak to peak change of transmittance, $f$ the spatial frequency. Under the irradiation of the light sheet, the photoluminescent molecules (or atoms) radiate via the photoluminescence processes. This radiation forms a luminescent gas sheet, which is imaged by a lens onto a two-dimensional detector array (CCD, CID, diode, etc.) with center line parallel to the $z$ axis. The electrical signal output is acquired and processed by a data processing system. Final results are displayed on the screen of a computer.

The field viewed by the two-dimensional detector array is A (Fig. 1), which is divided into a series of elementary areas $A_{i}(i=1,2,3, \ldots)$. Within each $A_{i}$ the gas flow is assumed to be parallel, uniform and steady, that means the temperature $T$, density $N$ and velocity component in the $x$ direction $\left(V_{x}\right)$ are constant within each $A_{i}$ for each period of data acquisition, but in different elementary areas and different times of data acquisition they might be different. If the gas flow does not satisfy this assumption then the $T, N$ and $V_{x}$ should be explained as the corresponding average values within each $A_{i}$ and each period of data acquisition.

The photoluminescence process can be divided into two stages: the excitation process and the decay process. By the excitation of the light sheet, the photoluminescent molecules (or atoms) in the lower level of the absorption transition are excited to the upper level, from which the excited molecules (or atoms) transit to the upper level of the luminescence transition via various kinds of processes [e.g. in the case of biacetyl, the intersystem crossing from ${ }^{1} A_{u}$ to ${ }^{3} A_{u}$ (Liu et al. 1988)], thus completing the excitation process. Then the population in the upper level of the luminescence transition decays via various processes (e.g. fluorescence, phosphorescence, quenching, dissociation, etc.). The intensity of the luminescence is directly proportional to the number density $N_{l}(x, t)$ of the upper level of the luminescence transition. The quantity $N_{l}(x, t)$ is affected by the excitation and the decay processes as well as the flow and the diffusion processes. Therefore $N_{l}(x, t)$ within a elementary area $A_{i}$ and one period of data acquisition obeys the following equation

$$
\begin{aligned}
\frac{\partial N_{l}(x, t)}{\partial t}= & D \frac{\partial^{2} N_{l}(x, t)}{\partial x^{2}}-V_{x} \frac{\partial N_{l}(x, t)}{\partial x}-\gamma N_{l}(x, t) \\
& +c\left(1-m_{s} \cos (2 \pi f x)\right)\left(1-m_{t} \cos (2 \pi \omega t)\right) \\
& t>0, \quad 0<x<\infty,
\end{aligned}
$$

where the first, second, third and fourth terms on the right side of the equal sign describe the diffusion, flow, decay and excitation processes, respectively. In Eq. (3) $D$ is the mutual diffusion coefficient of the primary gas and the photoluminescent gas. Based on a rigid spheres model, $D$ is given by (Present 1958)

$D=\frac{3}{8}\left(\frac{\pi k T}{2 m^{*}}\right) \frac{1}{N \pi d_{12}^{2}}$,

where

$d_{12}=\frac{1}{2}\left(d_{1}+d_{2}\right)$

$m^{*}=\frac{m_{1} m_{2}}{m_{1}+m_{2}}$.

$d_{1}, d_{2} ; m_{1} m_{2}$ are diameters and masses of the primary gas molecules (or atoms) and the photoluminescent molecules (or atoms), respectively. $k$ the Boltzmann constant. $\gamma$ in Eq. (3) is the reciprocal lifetime of the upper level of the luminescence transition. In general, $\gamma$ is a function of $T$ and $N$, but in the case of biacetyl $\gamma$ is independent of $N$ and can be given by an empirical formula (Liu et al. 1988)

$\gamma=\left(586+3.40 \times 10^{8} e^{-\frac{4.76 \times 10^{3}}{T}}\right) \mathrm{sec}^{-1}$,

where $T$ is in $K . c$ in Eq. (3) characterizes the excitation rate, which is affected by the absorption, the population of the lower level of the absorption transition, the rate constant of the transition from the upper level of the absorption transition to the upper level of the luminescence transition and the lifetimes of the upper levels of both the absorption and the luminescence transitions. The factor $c_{s}$ in Eq. (1) and the factor 1/2 in Eq. (2) are also included in $c$. In general, $c$ is also a function of $T$ and $N$. In the case of using biacetyl as the seed gas, $c$ is a linear function of $N$ (Liu et al. 1988).

The boundary and initial conditions of Eq. (3) are

$N_{l}(0, t)=N_{0}$

$N_{l}(x, 0)=N_{0}$, 
where $N_{0}$ is a constant. The right sides of the equal signs of Eqs. (8) and (9) must be equal to each other, otherwise the value of $N_{t}(0,0)$ will not be unique. Besides, the solution $N_{l}(x, t)$ must be finite to keep itself unique, i.e. the following condition must be satisfied

$\left|N_{l}(x, t)\right|<M \quad M=$ Constant.

\section{Solutions}

For solving Eq. (3) with the boundary and initial conditions (8) and (9) define a new variable

$$
M(x, t)=e^{-\alpha x-\beta t}\left(N_{l}(x, t)-N_{0}\right)
$$

where

$$
\alpha=\frac{V_{x}}{2 D}
$$

$\beta=-\frac{V_{x}^{2}}{4 D}-\gamma$

Using Eqs. (3), (8)-(12), the equation satisfied by $M(x, y)$, and corresponding boundary and initial conditions are given by

$$
\begin{aligned}
\frac{\partial}{\partial t} M(x, t)= & D \frac{\partial^{2}}{\partial x^{2}} M(x, t)+e^{-\alpha x-\beta t} \\
& \cdot\left[c\left(1-m_{s} \cos (2 \pi f x)\right)\left(1-m_{t} \cos (2 \pi \omega t)-\gamma N_{0}\right]\right.
\end{aligned}
$$

$M(0, t)=0$

$M(x, 0)=0$.

The solution is

$$
\begin{aligned}
& M(x, t)= \frac{1}{2 \sqrt{\pi}} \int_{0}^{\infty} \int_{0}^{t} \frac{1}{\sqrt{D(t-\tau)}} \\
& \cdot\left\{e^{-\frac{(x-\xi)^{2}}{4 D(t-\tau)}}-e^{-\frac{(x+\xi)^{2}}{4 D(t-\tau)}}\right\} e^{-\alpha \xi-\beta \tau} . \\
& {\left[c\left(1-m_{s} \cos (2 \pi f \xi)\right)\left(1-m_{t} \cos (2 \pi \omega t)\right)-\gamma N_{0}\right] d \xi d \tau . }
\end{aligned}
$$

In the text below, two cases of this solution will be investigated. Case 1: $m_{t}=0, m_{s} \neq 0$, i.e. the case in which the intensity of the light source is invariant and the grating has a transmittance cosinusoidally varying in the $x$ direction. Case 2: $m_{s}=0, m_{t} \neq 0$, i.e. the case in which the transmittance of the grating is constant and the light source is cosinusoidally varying with time. The case $m_{s} \neq 0, m_{t} \neq 0$ is beyond the scope of this investigation.

\subsection{Case 1: $m_{t}=0, m_{s} \neq 0$}

Using the convolution theory of the Fourier transform, the following formulas (17) and (18) can be proved (see Ap- pendix)

$$
\begin{gathered}
\frac{\sqrt{\pi}}{2 \sqrt{D(t-\tau)}} \int_{0}^{\infty} e^{-\alpha \xi} \cos (2 \pi f \xi)\left[e^{-\frac{(x-\xi)^{2}}{4 D(t-\tau)}}-e^{\left.-\frac{(x+\xi)^{2}}{4 D(t-\tau)}\right] d \xi}\right. \\
=\int_{0}^{\infty}\left\{\frac{2 \pi f+\lambda}{\alpha^{2}+(2 \pi f+\lambda)^{2}}-\frac{2 \pi f-\lambda}{\alpha^{2}+(2 \pi f-\lambda)^{2}}\right\} \\
\cdot e^{-\lambda^{2} D(t-\tau)} \sin \lambda x d \lambda \\
\frac{\sqrt{\pi}}{4 \sqrt{D(t-\tau)}} \int_{0}^{\infty} e^{-\alpha \xi}\left(e^{-\frac{(x-\xi)^{2}}{4 D(t-\tau)}}-e^{\left.-\frac{(x+\xi)^{2}}{4 D(t-\tau)}\right)} d \xi\right. \\
=\int_{0}^{\infty} \frac{e^{-D(t-\tau) \lambda^{2}} \lambda \sin \lambda x}{\lambda^{2}+\alpha^{2}} d \lambda
\end{gathered}
$$

By Eqs. (10), (16), (17) and (18) $N_{l}(x, t)$ is given by

$$
\begin{aligned}
N_{l}(x, t)-N_{0}=\frac{1}{\pi} e^{\alpha x+\beta t}\left\{\int_{0}^{\infty} \frac{\sin \lambda x}{D \lambda^{2}-\beta}\left(e^{-\beta t}-e^{-D \lambda^{2} t}\right)\right. \\
\cdot\left[\frac{2 \lambda\left(c-\gamma N_{0}\right)}{\lambda^{2}+\alpha^{2}}-c m_{s}\left[\frac{2 \pi f+\lambda}{(2 \pi f+\lambda)^{2}+\alpha^{2}}\right.\right. \\
\left.\left.\left.-\frac{2 \pi f-\lambda}{(2 \pi f+\lambda)^{2}+\alpha^{2}}\right]\right] d \lambda\right\}
\end{aligned}
$$

Since $-\beta+D \lambda^{2} \geq \gamma$, for

$t \gg \frac{1}{\gamma} \equiv \Delta t$

using the approximation

$e^{-\beta t}-e^{-D \lambda^{2} t} \simeq e^{-\beta t}$

Eq. (19) becomes

$$
\begin{aligned}
N_{l}(x)-N_{0}= & \frac{e^{\alpha x}}{\pi} \int_{0}^{\infty} \frac{\sin \lambda x}{D \lambda^{2}-\beta}\left[\frac{2 \lambda\left(c-\gamma N_{0}\right)}{\lambda^{2}+\alpha^{2}}-c m_{s}\right. \\
& \left.\cdot\left[\frac{2 \pi f+\lambda}{(2 \pi f+\lambda)^{2}+\alpha^{2}}-\frac{2 \pi f-\lambda}{(2 \pi f-\lambda)^{2}+\alpha^{2}}\right]\right] d \lambda .
\end{aligned}
$$

It is worthy of note that, as one can see from Eq. (21) $N_{l}$ becomes independent of $t$ for $t \gg \Delta t$. For accomplishing the integration in Eq. (21), use the Fourier sine transform of the factor $\sin \lambda x /\left(D \lambda^{2}-\beta\right)$, resulting in

$$
\begin{aligned}
N_{l}(x)-N_{0}= & \Lambda^{\prime}-\Psi_{f}^{\prime} \cos \left(2 \pi f x-\Phi_{f}\right) \\
& -e^{(\alpha-\sqrt{-\beta / D) x}}\left\{A^{\prime}-\Psi_{f}^{\prime} \cos \Phi_{f}\right\}
\end{aligned}
$$

where

$$
\begin{aligned}
& A^{\prime}=\frac{c-\gamma N_{0}}{\gamma} \\
& \Psi_{f}^{\prime}=\frac{c m_{s}}{\sqrt{\left[\gamma+(2 \pi f)^{2} D\right]^{2}+\left(2 \pi f V_{x}\right)^{2}}} \\
& \Phi_{f}=t_{g}^{-1}\left(\frac{2 \pi f V_{x}}{\gamma+(2 \pi f)^{2} D}\right) .
\end{aligned}
$$


The subscript $f$ indicates that the spatial frequency $f$ is an adjustable parameter of $\Psi_{f}^{\prime}$ and $\Phi_{f}$. For

$x \gg \frac{1}{\sqrt{\frac{-\beta}{D}}-\alpha} \equiv \Delta x$

Eq. (22) reduces to

$N_{l}(x)-N_{0}=\Lambda^{\prime}-\Psi_{f}^{\prime} \cos \left(2 \pi f x-\Phi_{f}\right)$.

The intensity of the photoluminescence emitted from the elementary area $A_{i}$ and received by the two-dimensional detector is given by

$I(x, t)=c_{i} h \vee K_{l} N_{l}(x, t)$,

where $c_{i}$ is a constant related to the structure of the particular instruments used, $h v$ the photon energy emitted, $K_{l}$ the luminescence rate constant. For $t \gg \Delta t$ and $x \gg \Delta x$, Eq (28) becomes

$I(x)=\Lambda-\Psi_{f} \cos \left(2 \pi f x-\Phi_{f}\right)$

where

$A=\frac{c_{0}}{\gamma} \quad c_{0}=c c_{i} h v K_{l}$

$\Psi_{f}=\frac{c_{0} m_{s}}{\sqrt{\left[\gamma+(2 \pi f)^{2} D\right]^{2}+\left(2 \pi f V_{x}\right)^{2}}}$

Provided $t \gg \Delta t$ and $x \gg \Delta x$, from Eq. (29) the following conclusions can be drawn:

(A) The variable $I(x)$ is independent of $t$, i. e. the distribution of $I(x)$ in space does not vary with time, provided the time is long enough after switching the light source on.

(B) The variable $I(x)$ is a cosine function of $x$ with the same spatial frequency $f$ as the grating and phase shift $\Phi_{f}$ with respect to the phase of the grating.

(C) The parameters $A, \Psi_{f}$ and $\Phi_{f}$ can be attained simultaneously either by measuring them individually with various instruments or by recording the steady $I(x)$ signal and then separating them with appropriate data processing equipments.

(D) In general, $c_{0}, \gamma$ and $D$ are functions of $T$ and $N$. For example, in the case of $N_{2}$ seeded with biacetyl, $c_{0}$ is directly proportional to $N$ and insensitive to the variation of temperature (Liu et al. 1988); $\gamma$ and $D$ as functions of $T$ and $N$ have already been shown in Eqs. (4) and (7). There are now three Eqs. (30), (31) and (25) for three unknowns $T, N$ and $V_{x}$. Therefore with the measured values of $A, \Psi_{f}$ and $\Phi_{f}$ the variables $T, N$ and $V_{x}$ can be determined by solving the simultaneous algebraic Eqs. (30), (31) and (25), provided $c_{0}$, $\gamma$ and $D$ as functions of $T$ and $N$ are known. In general, the dependences of $c_{0}, \gamma$ and $D$ on $T$ and $N$ can be obtained in advance by theoretical analyses and/or experiments for static gas samples such as what has been done for biacetyl (Liu et al. 1988).
(E) Since the spatial frequency $f$ is a adjustable parameter, other ways to determine $T, N$ and $V_{x}$ are to measure $\Psi_{f}$ and $\Phi_{f}$ with different spatial frequencies. That means, in addition to the measured values of $\Lambda, \Psi_{f}, \Phi_{f}$, the three simultaneous equations can also be obtained by measuring $\Psi_{f_{1}}, \Psi_{f_{2}}, \Phi_{f_{1}}$ or $A, \Phi_{f_{1}}, \Phi_{f_{2}}$ or $\Psi_{f_{1}}, \Psi_{f_{2}}, \Psi_{f_{3}}$; and so on. Since $\Lambda, \Psi_{f}$ and $\Phi_{f}$ might be measured with different instruments, this implies that different experimental arrangements with different instrument combinations can be chosen for the measurements of $T, N$ and $V_{x}$. Besides, one can change the measured values of $\Psi_{f}$ and $\Phi_{f}$ by changing the spatial frequency $f$ to fit the most favorable measurement ranges of the instruments available. The adjustable $f$ provides a flexibility to meet different experimental conditions.

(F) The direction of $V_{x}$ can be easily determined from the sign of $\Phi_{f}$ [Eq. (25)].

(G) A numerical example of case 1 is given for $\mathrm{N}_{2}$ seeded with biacetyl. The spatial distribution of the intensity of the photoluminescence received by the two dimensional detector in Case 1 [Eqs. (28), (22)] is shown in Fig. 2. The dependences of the average value, amplitude of modulation and phase shift on $T, N, V_{x}$ and the adjustable parameter $f$ are shown in Figs. 3-5, respectively.

\subsection{Case 2: $m_{s}=0, m_{t} \neq 0$}

Using the Eq. (16) for $t \gg \Delta t, N_{l}(x, t)$ is given by

$$
\begin{aligned}
N_{l}(x, t)- & N_{0}=\frac{c-\gamma N_{0}}{\gamma}\left(1-e^{-(\sqrt{-\beta / D}-\alpha) x}\right)-\frac{2 c m_{t}}{\pi} \\
& \cdot e^{\alpha x} \sqrt{\Pi_{c}^{2}(x)+\Pi_{s}^{2}(x)} \cos \left(2 \pi \omega t-t g^{-1}\left(\frac{\Pi_{s}(x)}{\Pi_{c}(x)}\right)\right.
\end{aligned}
$$

where

$$
\begin{aligned}
& \Pi_{s}(x)=2 \pi \omega \int_{0}^{\infty} \frac{\lambda \sin \lambda x}{\lambda^{2}+\alpha^{2}} \frac{1}{\left(D \lambda^{2}-\beta\right)^{2}+(2 \pi \omega)^{2}} d \lambda \\
& \Pi_{c}(x)=\int_{0}^{\infty} \frac{\lambda \sin \lambda x}{\lambda^{2}+\alpha^{2}} \frac{D \lambda^{2}-\beta}{\left(D \lambda^{2}-\beta\right)^{2}+(2 \pi \omega)^{2}} d \lambda .
\end{aligned}
$$

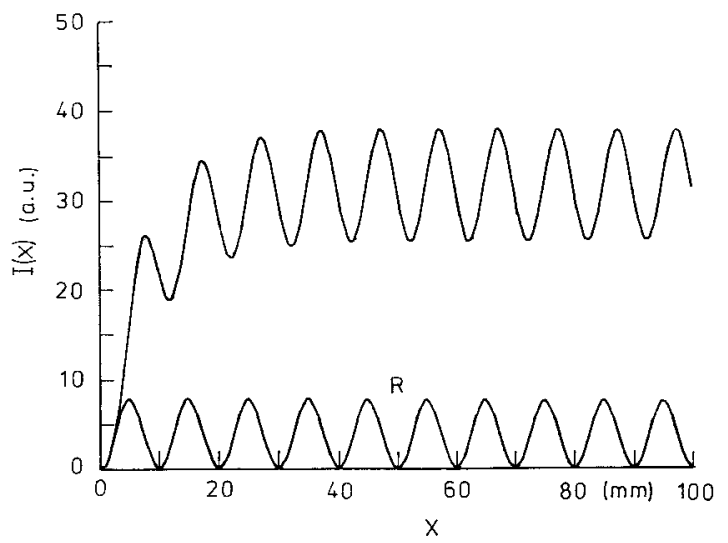

Fig. 2. Spatial distribution of the intensity of the photoluminescence received by the two-dimensional detector in Case $1 ; T=300 \mathrm{~K}$, $N=2 \times 10^{-3} \mathrm{~mol} / 1, V_{x}=5 \mathrm{~m} / \mathrm{s}, f=1001 / \mathrm{m}$, primary gas: $N_{2}$, seed gas: biacetyl, $m_{s}=1, m_{t}=0, N_{0}=0$; the spatial distribution of transmittance of the grating is shown as curve $R$ for reference 


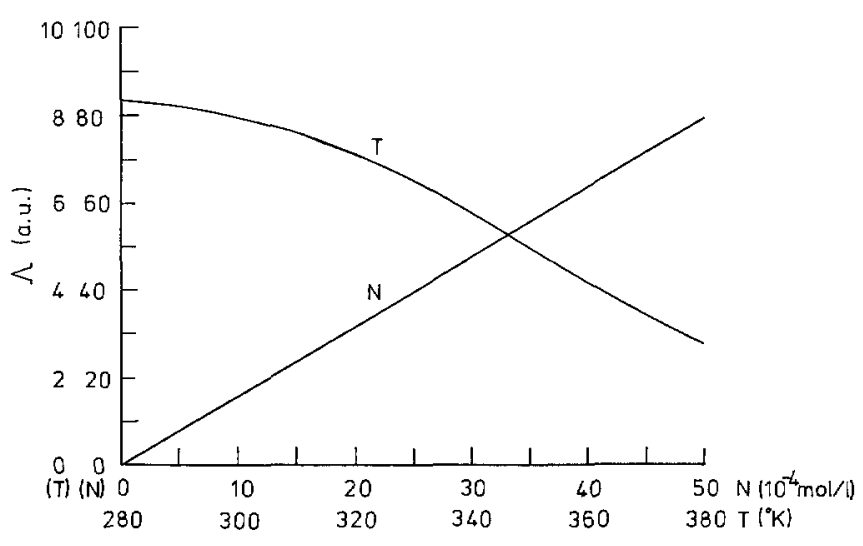

Fig. 3. Dependence of average value on temperature and density; $T=300 \mathrm{~K}, N=5 \times 10^{-4} \mathrm{~mol} / \mathrm{l}, V_{x}=10 \mathrm{~m} / \mathrm{s}, f=101 / \mathrm{m}$, unless it is the variable of the abscissa; primary gas: $N_{2}$, seed gas: biacetyl, $m_{s}=1, m_{t}=0$

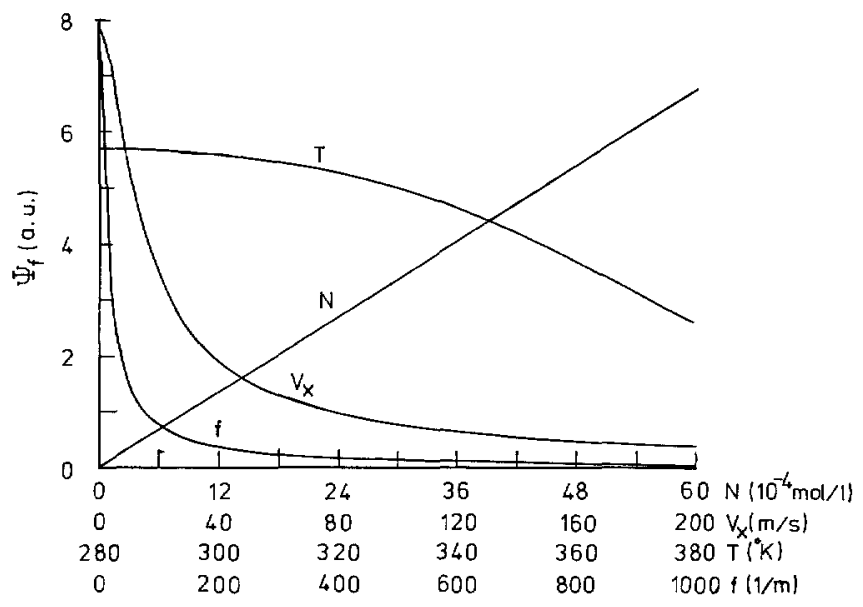

Fig. 4. Dependence of amplitude of modulation in Case 1 on temperature, density, $x$ component of velocity and spatial frequency: $T=300 \mathrm{~K}, N=5 \times 10^{-4} \mathrm{~mol} / 1, V_{x}=10 \mathrm{~m} / \mathrm{s}, f=101 / \mathrm{m}$, unless it is the variable of the abscissa; primary gas: $N_{2}$, seed gas: biacetyl, $m_{s}=1, m_{t}=0$

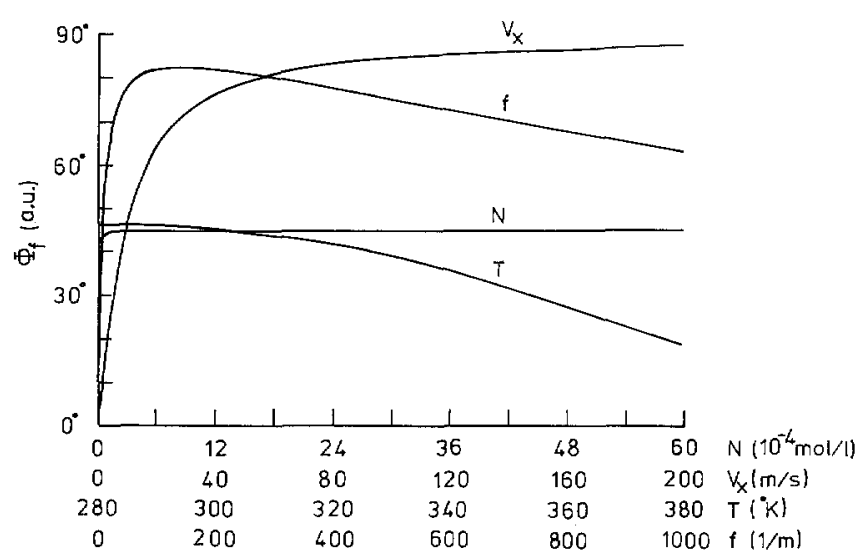

Fig. 5. Dependence of phase shift in Case 1 on temperature, density, $x$ component of velocity and spatial frequency: $T=300 \mathrm{~K}$, $N=5 \times 10^{-4} \mathrm{~mol} / \mathrm{l}, V_{x}=10 \mathrm{~m} / \mathrm{s}, f=101 / \mathrm{m}$, unless it is the variable of the abscissa; primary gas: $N_{2}$, seed gas: biacetyl, $m_{s}=1$, $m_{t}=0$
For accomplishing the integrations in Eqs. (33) and (34), use the Fourier sine transform of the function $\sin \lambda x /\left(\lambda^{2}+\alpha^{2}\right)$, resulting in

$$
\begin{aligned}
N_{l}(x, t)-N_{0}= & \frac{c-\gamma N_{0}}{\gamma}\left(1-e^{-(\sqrt{-\beta / D}-\alpha) x}\right)-\frac{c m_{t}}{\sqrt{\gamma^{2}+(2 \pi \omega)^{2}}} \\
& \cdot\left\{1-2 e^{\left(\alpha-A^{\prime}\right) x}\left[\cos t g^{-1}\left(\frac{2 \pi \omega}{\gamma}\right) \cos \left(B^{\prime} x-\eta\right)\right.\right. \\
& \left.+\sin t g^{-1}\left(\frac{2 \pi \omega}{\gamma}\right) \sin \left(B^{\prime} x+\eta\right)\right]+e^{2\left(\alpha-A^{\prime}\right) x} \\
& \cdot\left(1+\frac{1}{2}\left[\cos \left(2\left(B^{\prime} x-\eta\right)-\cos \left(2\left(B^{\prime} x-\eta\right)\right)\right]\right)\right\}^{1 / 2} \\
& \cdot \cos \left(2 \pi \omega t-t g^{-1}\right. \\
& \left.\cdot\left(\frac{2 \pi \omega+e^{\left(\alpha-A^{\prime}\right) x} \sqrt{(2 \pi \omega)^{2}+\gamma^{2}} \sin \left(B^{\prime} x+\eta\right)}{\gamma+e^{\left(\alpha-A^{\prime}\right) x} \sqrt{(2 \pi \omega)^{2}+\gamma^{2}} \cos \left(B^{\prime} x-\eta\right)}\right)\right)
\end{aligned}
$$

where

$$
\begin{aligned}
& A^{\prime}=\left(\frac{\sqrt{\left(\frac{-\beta}{D}\right)^{2}+\left(\frac{2 \pi \omega}{D}\right)^{2}}-\frac{\beta}{D}}{2}\right)^{1 / 2} \\
& B^{\prime}=\left(\frac{\left.\sqrt{\left(\frac{-\beta}{D}\right)^{2}+\left(\frac{2 \pi \omega}{D}\right)^{2}}+\frac{\beta}{D}\right)^{1 / 2}}{2}\right. \\
& \eta=\operatorname{tg}^{-1}\left(\frac{\frac{B^{\prime}}{\frac{\left(\alpha+A^{\prime}\right)^{2}+B^{\prime 2}}{\alpha+A^{\prime}}-\frac{B^{\prime}}{\left(\alpha-A^{\prime}\right)^{2}+B^{\prime 2}}}}{\frac{\alpha-A^{\prime}}{\left(\alpha+A^{\prime}\right)^{2}+B^{\prime 2}}+\frac{\left.\alpha-A^{\prime}\right)^{2}+B^{\prime 2}}{(\alpha-2}}\right)
\end{aligned}
$$

note that $A^{\prime} \geq \sqrt{\frac{-\bar{\beta}}{D}}$, in the case of $x \gg \Delta x, N_{l}$ reduces to

$N_{l}(t)-N_{O}=A^{\prime}-\Psi_{\omega}^{\prime} \cos \left(2 \pi \omega t-\Phi_{\omega}\right)$

where

$$
\begin{gathered}
\Psi_{\omega}^{\prime}=\frac{c m_{t}}{\sqrt{\gamma^{2}+(2 \pi \omega)^{2}}} \\
\Phi_{\omega}=t g^{-1}\left(\frac{2 \pi \omega}{\gamma}\right) .
\end{gathered}
$$

It is also worthy of note that from Eq. (39), for $t \gg \Delta t$ and $x \gg \Delta x, N_{l}$ becomes independent of $x$. The intensity of the luminescence emitted from the elementary area $A_{i}$ and received by the two-dimensional detector is given by

$I(t)=A-\Psi_{\omega} \cos \left(2 \pi \omega t-\Phi_{\omega}\right)$

where

$\Psi_{\omega}=\frac{\mathrm{c}_{0} m_{t}}{\sqrt{\gamma^{2}+(2 \pi \omega)^{2}}}$. 


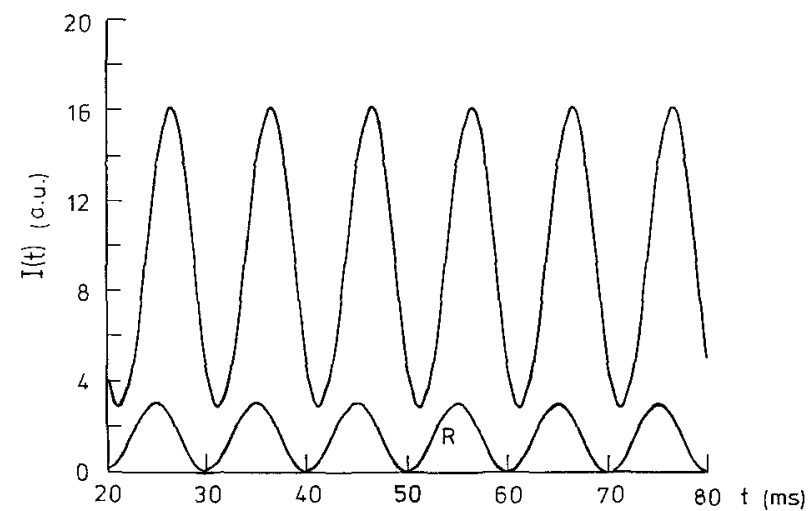

Fig. 6. Wave form of the intensity of the photoluminescence received by the two-dimensional detector in Case $2 ; T=300 \mathrm{~K}$, $N=6 \times 10^{-4} \mathrm{~mol} / 1, V_{x}=10 \mathrm{~m} / \mathrm{s}, \omega=100 \mathrm{~Hz}, x=0.1 \mathrm{~m}$, primary gas: $N_{2}$, seed gas: biacetyl, $m_{s}=0, m_{t}=1, N_{0}=0$; the waveform of the intensity of the light source is shown as curve $R$ for reference

Provided $t \gg \Delta t$ and $x \gg \Delta x$, from Eq. (42) the following conclusions can be drawn:

(A) The variable $I(t)$ is independent of $x$, i.e. in an area far enough from the end of the grating, $I(t)$ is a constant in space within each elementary area while varying cosinusoidally with time.

(B) The variable $I(t)$ is a cosine function of $t$ with the same frequency $\omega$ as the light source and phase shift $\Phi_{\omega}$ with respect to the phase of the light source.

(C) As one can see from Eq. (42), the velocity $V_{x}$ and diffusion coefficient $D$ have no effect on $I(t)$. This can be anticipated because: (a) according to the model, the flow is parallel, uniform and steady; (b) the transmittance of the grating is constant in the $x$ direction; (c) for $x \gg \Delta x$ the effect of the end of the grating can be neglected. There is no physical reason for the existence of the gradient of $N_{l}$, therefore the diffusion has no effect on $I(t)$. The motion of the gas continuously changes the part of the gas observed by the two-dimensional detector, but by the same reason as mentioned above, the photoluminescent signal received by the detector will not be changed by the motion, therefore $V_{x}$ has no effect on $I(t)$ either. The fact that neither $V_{x}$ nor $D$ has an effect on $I(t)$ makes the experimental principle of Case 2 applicable in flow fields with wide ranges of $V_{x}$ and $D$, but no information about $V_{x}$ can be acquired, consequently only two parameters - $T$ and $N$ can be measured. Note that the spatial resolution in Case 2 is still affected by $V_{x}$ and $D$ (Sect. 6).

(D) As in Case 1, the parameters $A, \Psi_{\omega}$ and $\Phi_{\omega}$ can be obtained simultaneously either by measuring them individually with various instruments or by recording the $I(t)$ signal and then separating them with appropriate data processing equipment.

(E) There are three Eqs. (30), (41), and (43) for only two unknowns $T$ and $N$. Provided the dependences of $c_{0}$ and $\gamma$ on $T$ and $N$ are known by theoretical analyses and/or experiments, $T$ and $N$ can be determinated by solving the simulta-

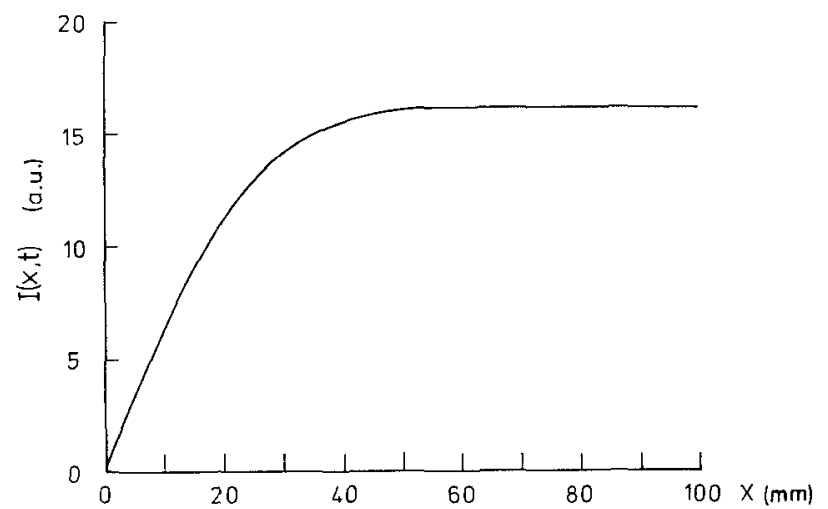

Fig. 7. Spatial distribution of the intensity of the photoluminescence received by the two-dimensional detector in Case $2 ; T=300 \mathrm{~K}$, $N=6 \times 10^{-4} \mathrm{~mol} / 1, V_{x}=10 \mathrm{~m} / \mathrm{s}, \omega=100 \mathrm{~Hz}, t=46 \mathrm{~ms}$, primary gas: $N_{2}$, seed gas: biacetyl, $m_{s}=0, m_{t}=1, N_{0}=0$

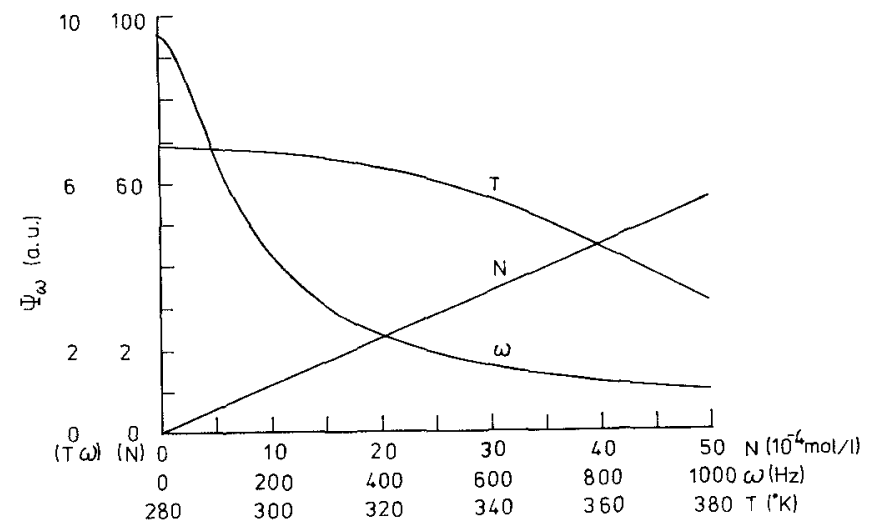

Fig. 8. Dependence of amplitude of modulation in Case 2 on temperature, density, and frequency: $T=300 \mathrm{~K}, N=6 \times 10^{-4} \mathrm{~mol} / \mathrm{l}$, $\omega=100 \mathrm{~Hz}$, unless it is the variable of the abscissa; primary gas: $N_{2}$, seed gas: biacetyl, $m_{s}=0, m_{t}=1$

neous equations consisting of any two of the Eqs. (30), (41), and (43). The third one could serve for checking purpose.

(F) Since the frequency $\omega$ is a adjustable parameter. The two simultaneous equations can also be obtained by measuring values of $\Psi_{\omega_{1}}, \Psi_{\omega_{2}}$ or $\Phi_{\omega_{1}}, \Phi_{\omega_{2}}$.

(G) A numerical example of Case 2 is given for $\mathrm{N}_{2}$ seeded with biacetyl. The wave form and the spatial distribution of the intensity of the photoluminescence received by the twodimensional detector in Case 2 [Eqs. (28) and (35)] are shown in Figs. 6 and 7, respectively. The dependences of the amplitude of modulation $\Psi_{\omega}$ and phase shift $\Phi_{\omega}$ on $T, N$ and the adjustable parameter $\omega$ are shown in Figs. 8 and 9, respectively. The average value $A$ in Case 2 is the same as in Case 1 .

\section{Solution of Case 1 with two velocity components}

So far we have assumed that the velocity has only one component $V_{x}$. In Sects. 4 and 5 we shall investigate the case in 


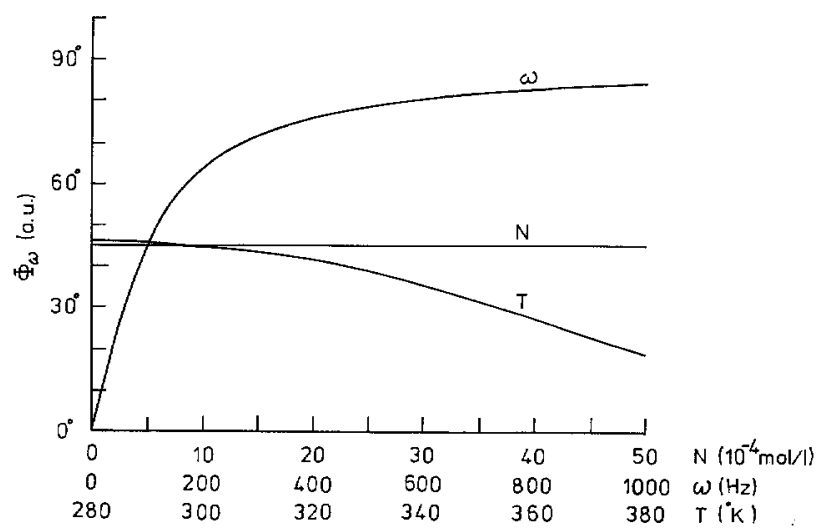

Fig. 9. Dependence of phase shift in Case 2 on temperature, density, and frequency: $T=300 \mathrm{~K}, N=6 \times 10^{-4} \mathrm{~mol} / 1, \omega=100 \mathrm{~Hz}$, unless it is the variable of the abscissa; primary gas: $N_{2}$, seed gas: biacetyl, $m_{s}=0, m_{t}=1$

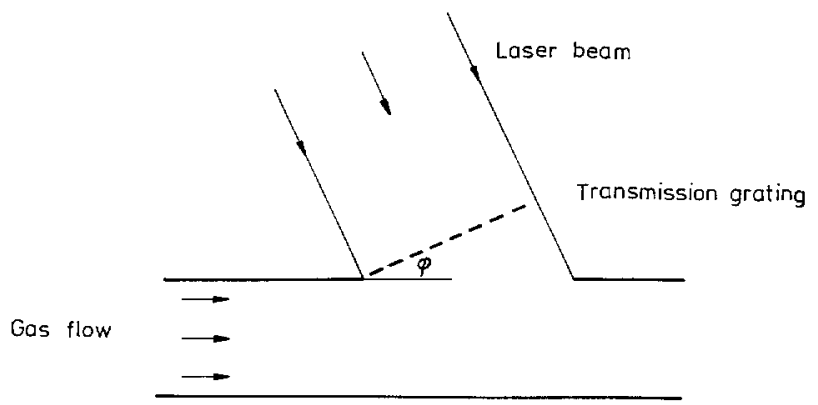

Fig. 10. Experiment with turned transmission grating for two velocity component flows

which $m_{t}=0, m_{s} \neq 0$ (Case 1), but the velocity has two components $V_{x}$ and $V_{y}$. We assume that $N_{l}$ has already been in steady state, i.e. $\frac{\partial}{\partial t} \mathbf{N}_{l}=0$. Besides, diffusion in the $y$ direc-

tion will be neglected since there is not any physical reason to expect that a gradient of $N_{l}$ will exist in the $y$ direction because the intensity of the excitation light is independent of $y$ and we only pay attention to the area where both $x$ and $y$ are far enough from the boundaries so that the effects of boundaries are negligible. Therefore $N_{l}(x, y)$ obeys the following equation and boundary conditions

$$
\begin{aligned}
& D \frac{\partial^{2} N_{l}(x, y)}{\partial x^{2}}-V_{x} \frac{\partial N_{l}(x, y)}{\partial x}-V_{y} \frac{\partial N_{l}(x, y)}{\partial y}-\gamma N_{l}(x, y) \\
& +c\left(1-m_{s} \cos (2 \pi f x)\right)=0 \\
& N_{l}(x, 0)=N_{0} \\
& N_{l}(0, y)=N_{0} \text {. }
\end{aligned}
$$

From a mathematical point of view, Eq. (44) is the same as Eq. (3) in Case 1. If $y / V_{y}$ is substituted for $t$ in Eq. (3), then Eq. (3) becomes Eq. (44). Therefore the solution of Eq. (44) with boundary conditions (45) and (46) can be solved easily. Ne- glecting the terms which exponentially decay with $y$,

$I(x, y)=A-\Psi_{f} \cos \left(2 \pi f-\Phi_{f}\right)$.

It is not surprising that $I(x, y)$ [Eq. (47)] is just the same as $I(x)$ [Eq. (29)]. As mentioned above, $I(x)$ is independent of $t$; therefore the substitution of $t$ for $y / V_{y}$ will cause no change in the solution. As a result, $I(x, y)$ is independent of $y / V_{y}$. The conclusion drawn from Eq. (47) is that in the case of two velocity components the existence of $V_{y}$ has no effect on the photoluminescent signal received by the detector, but $V_{y}$ can not be measured by the method of Case 1 .

\section{Measurement of two velocity components}

In order to find a way to measure both $V_{x}$ and $V_{y}$, we investigate the solution of Case 1 with a transmission grating turned to an angle $\varphi$ around the point $x=0$ (Fig. 10). For this case $I(x, y)$ can be obtained simply from Eq. (47) with a . coordinate transformation; the result is given by

$$
\begin{aligned}
& I(x, y)=\frac{c_{0}}{\gamma}-c_{0} m_{s} \\
& \frac{\cos \left(2 \pi f(x \cos \varphi+y \sin \varphi)-t g^{-1}\left(\frac{2 \pi f\left(V_{x} \cos \varphi+V_{y} \sin \varphi\right)}{\gamma+(2 \pi f)^{2} D}\right)\right)}{\sqrt{\left[\gamma+(2 \pi f)^{2} D\right]^{2}+\left[2 \pi f\left(V_{x} \cos \varphi+V_{y} \sin \varphi\right)\right]^{2}}}
\end{aligned}
$$

By solving the three simultaneous equations as mentioned above, only $T, N$ and $V_{\varphi}=V_{x} \cos \varphi+V_{y} \sin \varphi$ can be obtained. In order to determine $V_{x}$ and $V_{y}$ an additional experiment has to be carried out. For example, one can do this experiment twice with two different angles $\varphi_{1}$ and $\varphi_{2}$, obtaining two measured velocity values $V_{\varphi_{1}}$ and $V_{\varphi_{2}}$

$V_{\varphi_{1}}=V_{x} \cos \varphi_{1}+V_{y} \sin \varphi_{1}$

$V_{\varphi_{2}}=V_{x} \cos \varphi_{2}+V_{y} \sin \varphi_{2}$.

With known parameters $\varphi_{1}$ and $\varphi_{2}, V_{x}$ and $V_{y}$ can be determined by solving the simultaneous equations (49) and (50).

\section{Temporal and spatial resolutions}

The temporal and spatial resolutions depend on several factors: (a) The temporal and spatial resolutions of the particular instruments chosen for measurements, which will not be discussed here. (b) The temporal and spatial periods of the light source and the transmission grating. Obviously the temporal and spatial resolutions can not be smaller than one period of the light source and transmission grating, respectively. This factor will not be discussed here either, since there are various techniques which can always provide appropriate temporal and spatial modulations. (c) The factors which are determined by the measurement principles adopted will be investigated here. As mentioned above the inequality $t \gg \Delta t$ [Eq. (20)] describes how long it should take 


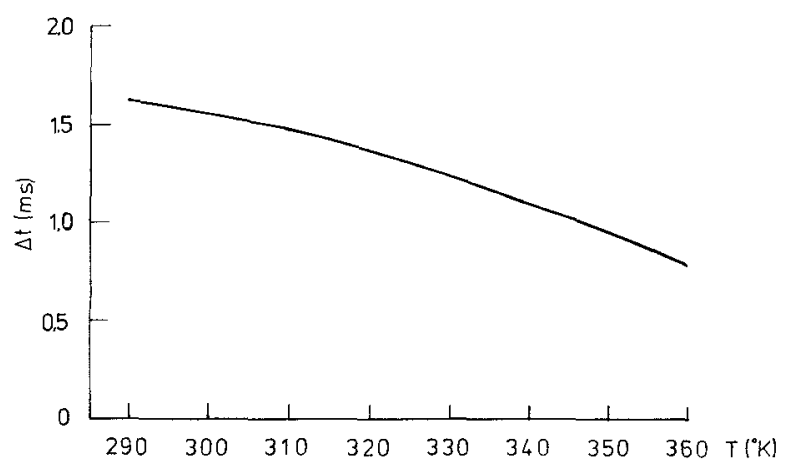

Fig. 11. Dependence of temporal resolution on temperature; primary gas: $N_{2}$, seed gas: biacetyl

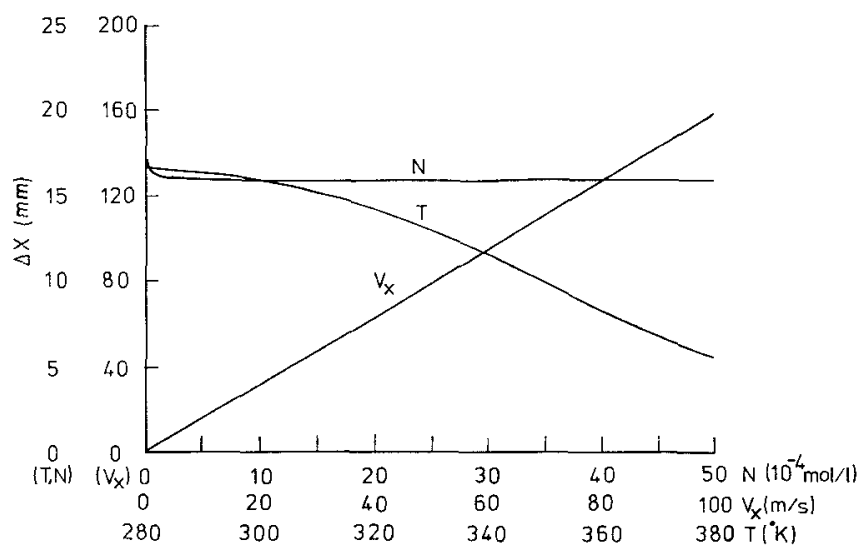

Fig. 12. Dependence of the spatial resolution on temperature, density and $x$ component of velocity; $T=300 \mathrm{~K}, N=6 \times 10^{-4} \mathrm{~mol} / 1$, $V_{x}=10 \mathrm{~m} / \mathrm{s}$, unless it is the variable of the abscissa; primary gas: $N_{2}$, seed gas: biacetyl

for an elementary area starting from a state of $N_{l}=N_{0}$ to change to another state in which $N_{l}$ varies cosinusoidally with $t$ or $x$. Similarly the inequality $x \gg \Delta x$ [Eq. (26)] describes how far apart two adjacent elementary areas $A_{i}$, in which $N_{l}=N_{0}$, and $A_{j}$, in which $N_{l}$ varies cosinusoidally with $t$ or $x$, should be. $\Delta t$ and $\Delta x$ can serve as an approximate measure of the temporal and spatial resolutions, respectively, since measurements with the principles described in Sect. 2 and 3 for two events with time difference less than $\Delta t$ or for two points with a distance less then $\Delta x$ are impossible. The time difference and the distance should be approximately 7 times larger than the values of $\Delta t$ and $\Delta x$, respectively, to keep the relative error less than $1 \%$. Note that $\Delta t$ is a function of $\gamma$ and $\Delta x$ of $\gamma, D$, and $V_{x}$. Therefore the temporal and spatial resolutions $\Delta t$ and $\Delta x$ depend on the photoluminescent material chosen as well as the state of the gas flow.

Numerical examples are given for $N_{2}$ seeded with biacetyl. The dependences of $\Delta t$ on $T$ and $\Delta x$ on $T, N$ and $V_{x}$ are shown in Figs. 11 and 12.

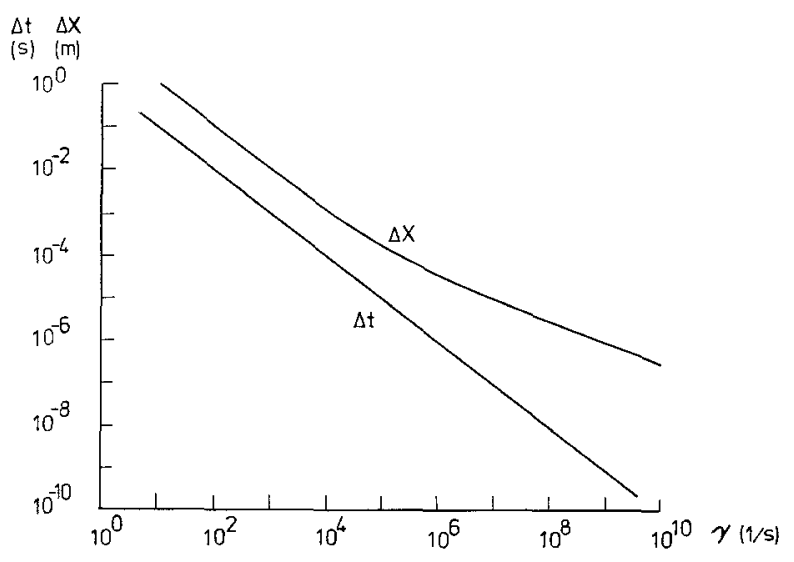

Fig. 13. Dependence of average value, amplitudes of modulations and phase shifts in Case 1 and 2 on reciprocal lifetime; $T=300 \mathrm{~K}$, $N=6 \times 10^{-4} \mathrm{~mol} / 1, \quad V_{x}=1000 \mathrm{~m} / \mathrm{s}, \quad f=101 / \mathrm{m}, \quad \omega=10^{6} \mathrm{~Hz}$, $D=7.9 \times 10^{-4} \mathrm{~m}^{2} / \mathrm{s}$

$\wedge \underset{(a . U)}{\Psi_{f}} \Psi_{\omega} \Phi_{t} \Phi_{\omega}$

$10^{10} 1001000$

$10^{8} 80800$
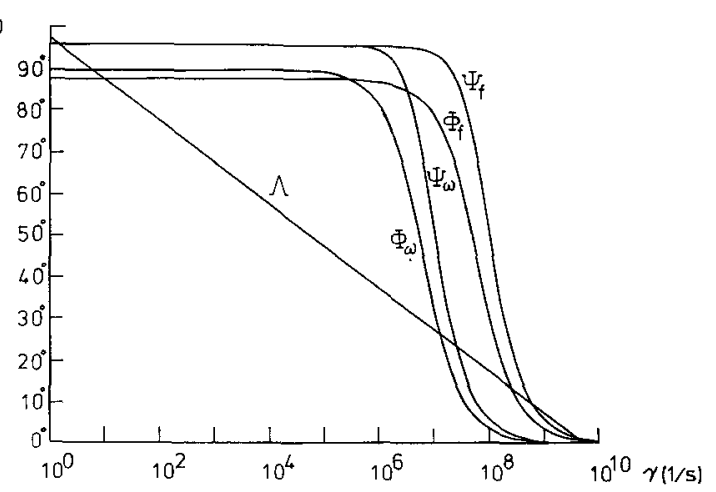

$10^{0} 0$

Fig. 14. Dependence of temporal and spatial resolutions on reciprocal lifetime; $T=300 \mathrm{~K}, N=6 \times 10^{-4} \mathrm{~mol} / 1, V_{x}=10 \mathrm{~m} / \mathrm{s}$, $D=7.9 \times 10^{-4} \mathrm{~m}^{2} / \mathrm{s}$

\section{Discussion}

(A) Besides biacetyl, which is used as an example in this paper, many gaseous photoluminescent materials including fluorescent and phosphorescent gases could be considered as seed gas provided the decay of their photoluminescence is single-exponential. This provides a flexibility to meet different experimental demands in different measurement ranges of $T, N$ and $V_{x}$ by properly choosing the seed gas.

The most important physical character of the seed material is the lifetime $(1 / \gamma)$, which varies from $\gtrsim 10^{-3} \mathrm{sec}$. (phosphorescence) to $10^{-6}-10^{-8} \mathrm{sec}$. (fluorescence). Compared with the lifetime, the mutual diffusion coefficient $D$ does not change very much for different photoluminescent materials. Numerical examples for showing the effects of lifetime on $A$, $\Psi_{f}, \Phi_{f}, \Psi_{\omega}, \Phi_{\omega}, \Delta t$ and $\Delta x$ are given in Figs. 13 and 14, in which $D$ is assumed to be constant while $\gamma$ varies several orders of magnitude. Fig. 14 implies that photoluminescent gases with large $\gamma$ (short lifetime) should be chosen for measurements with high temporal and spatial resolutions. 
(B) Since the seed materials are gaseous, the particle lag problem as encountered in Doppler velocimetry can be avoided to a great extent.

(C) By using experimental dependences of $\gamma$ on $T$ and $N$, the quenching effect can be taken into account.

(D) The dependences of $c_{0}, \gamma$ and $D$ on $T$ and $N$ could be measured in conventional ways. But the following method is perhaps more convenient. Using the same experimental setup as used in flow measurements, substitute a static sample cell with controllable temperature and density for the gas flow. Again the parameters $c_{0}, \gamma$ and $D$ can be determined by solving three simultaneous equations with various combinations of the measured values of the average value $A$, amplitudes of modulation $\left(\Psi_{f}\right.$ and $\left.\Psi_{\omega}\right)$ and phase shifts $\left(\Phi_{f}\right.$ and $\left.\Phi_{\omega}\right)$, e.g., $\Psi_{\omega}, \Phi_{\omega}, \Psi_{f} ; \Psi_{f_{1}}, \Psi_{f_{2}}, \Phi_{\omega}$ etc.

(E) The temporal modulation can be achieved by mechanical and/or electric means for low modulation frequency or by acousto-optic modulators and electro-optic modulators for high modulation frequency. The spatial modulation can be achieved by transmission gratings or by crossed laser beams. In principle, both coherent and incoherent light sources can be used. Thus besides the dye laser, many incoherent spectral lamps can be considered as inexpensive light sources. For example, in case of biacetyl as seed gas, a Mercury-Gallium lamp is a good light source. To avoid the possible influence of Doppler effect, broad bandwidth light source should be used in the case of photoluminescent gas with narrow absorption bandwidth.

(F) According to the measurement principles described in this paper, very precise or powerful instruments are not needed, therefore the costs are expected to be relatively low.

(G) There is a type of instrument for lifetime measurement called phase-shift and amplitude-modulated technique (PSAMT) (Yguerabide and Yguerabide 1984), in which the sample is excited with a sinusoidally modulated light source and the lifetime is determined from the phase shift or amplitude of modulation. PSAMT is just Case 2 of the method described in this paper with $V_{x}=0$, remembering that $\gamma$ is the reciprocal lifetime. The theoretical result of the intensity of photoluminescence received by detector [Eq. (42)] also agrees with that in PSAMT (Ware 1971). The PSAMT has been used successfully since 1927 (Worth and Chen 1988; Bright et al. 1986; Keating-Nakamoto et al. 1987) and several PSAMT instruments are commercially available (e.g. GREG-200, I.S.S.; 48000S Instruments). Although the PSAMT is only for lifetime measurement and has nothing to do with the flow measurement, its success implies that a similar method in flow measurement might be promising. Besides this, the abundant experience in using PSAMT (Briks and Munro 1967; Ware 1971) is worth consideration.

\section{Summary}

Simultaneous temperature, density and velocity measurements should be possible by measuring the average value, amplitude of modulation and phase shift of the photoluminescence excited by a temporally or spatially modulated light source. The potential advantages of this method are: (a) simultaneous measurements of $T, N$ and $V_{x}$; (b) easy determination of the direction of $V_{x}$; (c) applicability to many gaseous photoluminescent materials; (d) highly flexible experimental arrangements; (e) suitability for two dimensional measurement; (f) possibilities of avoiding the particle lag problem and taking the quenching effect into account and $(\mathrm{g})$ relatively low costs.

\section{Acknowledgements}

The authors thank Prof. Zheng Zhe-min, Prof. Wu Cheng-kang and Prof. Xie Bo-min for their fruitful discussions. The authors thank National Natural Science Foundation of China and The Third World Academy for their support.

\section{Appendix}

Derivation of Eqs. (17) and (18)

The convolution theorem of the Fourier transform is (Goodman 1968)

$\int_{-\infty}^{+\infty} \bar{f}(\lambda) \bar{g}(\lambda) e^{-i \lambda x} d \lambda=\int_{-\infty}^{+\infty} g(s) f(x-s) d s$

where $\bar{f}(\hat{\imath})$ and $\bar{g}(\lambda)$ are the Fourier transforms of the functions $f(\xi)$ and $g(\xi)$, e.g.

$\bar{f}(\lambda)=\frac{1}{\sqrt{2 \pi}} \int_{-\infty}^{+\infty} f(\xi) e^{-i \lambda \xi} d \xi$.

From Eqs. (51) and (52) it can be obtained that

$\int_{0}^{\infty} f_{s}(\lambda) g_{c}(\lambda) \sin \lambda x d \lambda=\frac{1}{2} \int_{0}^{\infty} f(s)[g(|x-s|)-g(x+s)] d s$

where

$$
\begin{aligned}
& f_{s}(\lambda)=\sqrt{\frac{2}{\pi} \int_{0}^{\infty} f(\xi) \sin \lambda \xi d \xi} \\
& g_{c}(\lambda)=\sqrt{\frac{2}{\pi}} \int_{0}^{\infty} g(\xi) \cos \lambda \xi d \xi
\end{aligned}
$$

Let the functions $f(\xi)$ and $g(\xi)$ in Eqs. (53)-(55) be

$f(\xi)=e^{-\alpha \xi}$
$g(\xi)=e^{-\frac{\xi^{2}}{4 D(t-\tau)}}$

or

$f(\xi)=e^{-\alpha \xi} \cos (2 \pi f \xi)$

$g(\xi)=e^{-\frac{\xi^{2}}{4 D(t-\tau)}}$

then Eqs. (18) and (17) can be proved, respectively. 


\section{References}

Blint, R. J.; Bechtel, J. H.; Stephensen, D. A. 1980: Carbon dioxide concentration and temperature in flames by Raman spectroscopy. J. Quant. Spectrosc. Radiat. Transfer. 23, 89-94

Bram, R. D.; Seiler, E. T.; LaRue, J. C.; Samnelsen, G. S. 1983: Instantaneous two-component laser anemometry and temperature measurements in a complex flow model combustor. AIAA Paper 83-0334

Bright, F. V.; Daniel, D. A.; Wilson, A.; Hieftie, G. M. 1986: Determination of subnanosecond fluorescence lifetime with a UHF television tuner and a cw or mode-locked laser. Appl. Spectro. $40,683-687$

Briks, J. B.; Munro, I. H. 1967: The fluorescence lifetimes of aromatic molecules. In: Progress in reaction kinetics (ed. Poter, G.). Vol. 4, pp 239-303. Oxford: Pergamon Press

Cheng, S.; Zimmermann, M.; Miles, R. B. 1983 a: Supersonic-nitrogen flow-field measurements with the resonant Doppler velocimeter. Appl. Phys. Lett. 43, 143-145

Cheng, S..; Zimmermann, M.; Miles, R. B. 1983 b: Mean square velocity, temperature and pressure fluctuation by LIF. Phys. Fluid. 26, 874-877

Fujii, S.; Gomi, M.; Equchi, K. 1983: A remote laserprobe system for velocity and temperature measurements. J. Fluids Eng. 105, $128-133$

Fujii, S.; Gomi, M.; Equchi, K.; Yamayuchi, S.; Jin, L. 1984: Time resolved $\mathrm{LDV}$ and CARS measurements in a premixed reacting flow. Combust. Sci. Technol. 36, 211-226

Goodman, J. W. 1968: Introduction to Fourier optics. New York: McGraw-Hill

Goss, L. P.; Trump, D. D.; Roquemore, W. M. 1984: Simultaneous CARS and LDA measurements in a turbulent flame. AIAA J. $84-1458$

Goss, L. P.; Trump, D. D.; Roquemore, W. M. 1988: Combined CARS/LDA instrument for simultaneous temperature and velocity measurements. Exp. Fluids 6, 189-198

Gross, K. P.; McKenzie, R. L.; Logen, P. 1987: Measurements of temperature, density, pressure and their fluctuations in supersonic turbulent using laser induced fluorescence. Exp. Fluids 5, $372-380$

Keating-Nakamoto, S. M.; Cherck, N.; Lakowicz, J. R. 1987: Resolution of multicomponent fluorescence emission by phase sensitive detection at multiple modulation frequencies. Anal. Chem. $59,271-278$
Liu, Jian-bang; Pan Qi; Liu Chang-sheng; Shi Jie-rong 1988: Principles of flow field diagnostics by laser induced biacetyl phosphorescence. Exp. Fluids 6, 505-513

Luck, K. C.; Thielen, W. 1978: Measurements of temperature and $\mathrm{OH}$ concentrations in a lean methane-air flame using high resolution laser absorption spectroscopy. J. Quant. Spectrosc. Radiat. Transfer. 20, 71-79

Miles, R. B. 1975: Resonant Doppler velocimeter. Phys. Fluids 18, $751-752$

Miles, R. B.; Udd, E.; Zimmermann, M. 1978: Quantitative flow visualization in sodium vapour seeded hypersonic helium. Appl. Phys. Lett. 32, 317-319

Present, R. D. 1958: Kinetic theory of gases. New York: McGrawHill

Rose, A.; Gupta, R. 1986: Application of photothermal and photoacoustic techniques to sooting flames. Opt. Commun. 56, $303-$ 308

Smith, J. A.; Driscoll, J. F. 1975: The electron-beam fluorescence technique for measurements in hypersonic turbulent flows. J. Fluid Mech. 72, 695-719

Smith, J. R 1980 a: Temperature and density measurements in an engine by pulsed Raman spectroscopy. SAE paper 800137

Smith, J. R. 1980 b: Instantaneous temperature and density by spontaneous Raman scattering in a piston engine. AIAA Paper $80-1359$

Stephered, I. G.; Moss, J. B.; Bray, K. N. C. 1982: Turbulent transport in a combined premixed flame. In: 19th Symposium (International) on combustion. (eds. Krier, H.; Dabora, E.). pp. 423-431. Ann Arbor/MI: Edwards Brothers

Ware, W. R. 1971: Transient luminescence measurements. In: Creation and detection of the excited state (ed. Lamola, A. A.). Vol. 1, part 1, pp 213-300. New York: Marcel Dekker

Worth, W. J.; Chen, S-H 1988: Phase-resolved subnanosecond spectroscopy using the beat frequencies from mode-locked laser. Appl. Spectroscopy 42, 483-486

Yquerabide, J.; Yquerabide, E. 1984: Nanosecond fluorescence spectroscopy. In: Optical techniques in biological research (ed. Rousseau, D. L.). pp 181-287. Orlando, Florida: Academic Press

Zimmermann, M.; Miles, R. B. 1980: Hypersonic-helium-flow-field measurements with the resonant Doppler velocimeter. Appl. Phys. Lett. $37,885-887$

Received May 30, 1989 